Grupos interactivos

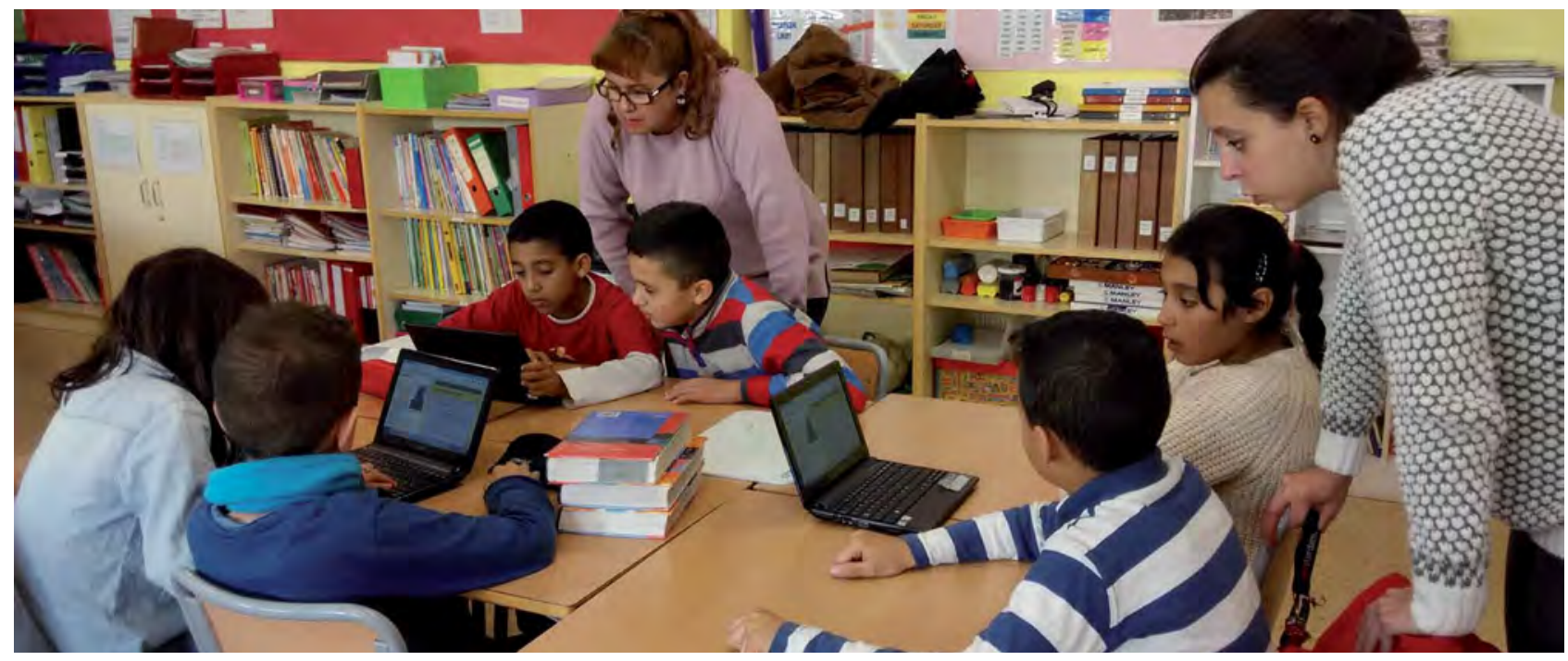

Interacciones que aumentan

el aprendizaje y la solidaridad

Los grupos interactivos son la forma de organización del aula que da los mejores resultados en la actualidad en cuanto a la mejora del aprendizaje y la convivencia. A través de los grupos interactivos, se multiplican y diversifican las interacciones a la vez que aumenta el tiempo de trabajo efectivo. Se caracterizan por ser una organización inclusiva del alumnado en la que se cuenta con la ayuda de más personas adultas además del profesor o profesora responsable del aula.

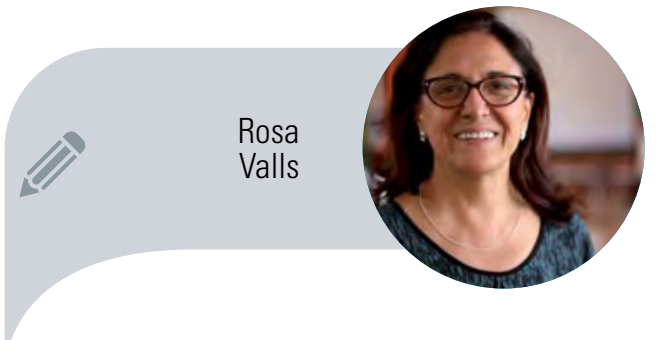

Departamento de Teoría e Historia de la Educación, Universidad de Barcelona rosavalls@ub.edu

Nataly Buslón Departamento de Teoría Sociológica, Filosofía del Derecho y Metodología de las Ciencias Sociales, Universidad de Barcelona nataly.buslon@ub.edu

Isabel López Departamento de Comunicación y Educación, Universidad Loyola Andalucía

ilopez@uloyola.es 


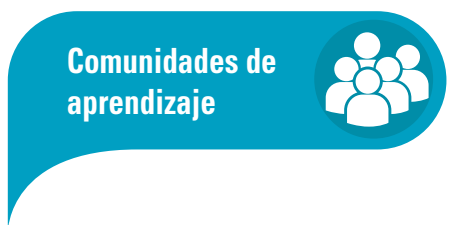

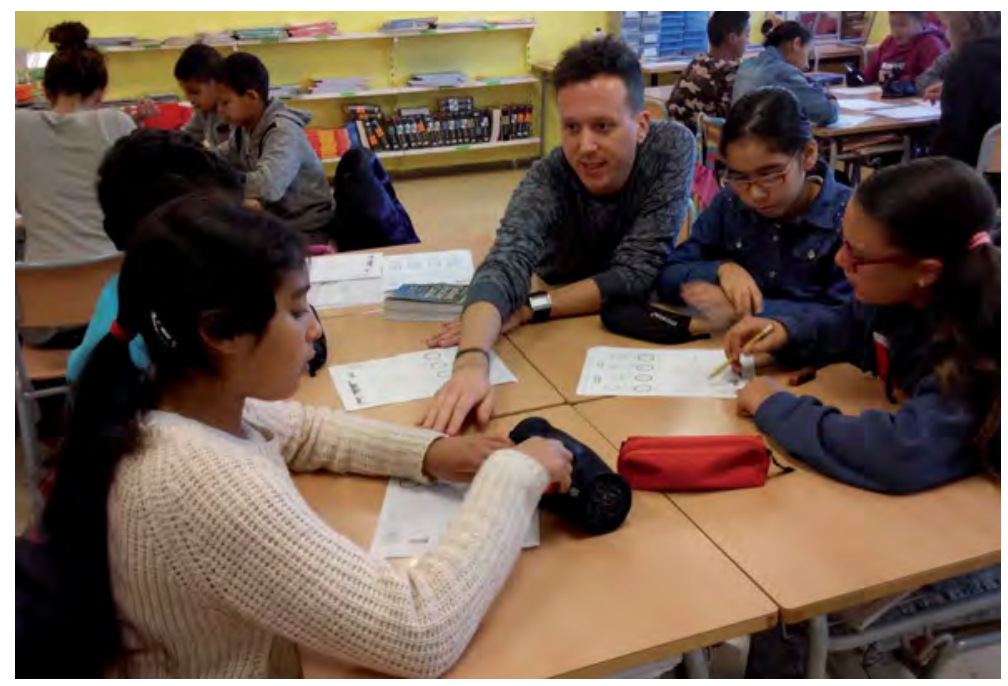

Los grupos interactivos son una actuación educativa de éxito (AEE), identificada en el proyecto "INCLUD-ED" (2006-2011), única investigación en ciencias sociales que la Comisión Europea seleccionó en el 2011 entre las diez de mayor éxito en investigaciones científcas en Europa (European Commission, 2011). INCLUD-ED realizó un análisis de estrategias educativas que contribuían a superar las desigualdades y fomentaban la cohesión social, marcando el éxito en la educación en un modelo alternativo que brinda un cambio a la realidad presente. Las AEE se orientan a una educación inclusiva, que logra reducir el absentismo y el abandono escolar temprano generando altas expectativas para todo el alumnado y oportunidades de empleo y participación social.

Las AEE son universales y transferibles a cualquier contexto educativo y social, junto a las comunidades de aprendizaje tienen como objetivo la transformación social y educativa, mediante las interacciones y la participación de la comunidad (DíEZ \& FLECHA, 2010). La apertura a distintos agentes en las aulas facilita nuevos espacios de diálogo, intercambio y solidaridad, que impulsan los procesos de aprendizaje de los niños potenciando a quienes presentan dificultades y se encuentran en una situación socioeconómica más vulnerable.
Los grupos interactivos (GI) consisten en organizar las clases en pequeños grupos heterogéneos en rendimiento, género, cultura, etc.... de alumnos, cada grupo a cargo de una persona adulta voluntaria que facilita la interacción, el diálogo y el aprendizaje. El docente coordina las actividades y proporciona apoyo cuando es necesario. Son grupos de 4 o 5 alumnos, heterogéneos, con cambio de actividades cortas cada 15 o 20 minutos. La interacción es un factor clave en la actividad; el momento del cambio de actividad influye y genera un aumento de la motivación, involucrándolos en el trabajo y ayuda al alumnado a enfrentar nuevas situaciones y estimula su aprendizaje. Lo importante en cada momento es promover las interacciones con altas expectativas que marcan el éxito de la actividad.

Al contrario de la práctica de agrupación por aptitudes que segrega a los estudiantes de acuerdo a su capacidad y también en clases de recuperación, y prestación de apoyo separados del grupo (Molina y Ríos, 2010), los GI apuntan a la inclusión del alumnado para la ayuda mutua y mejora de los resultados en beneficio del alumnado en su totalidad. El aprendizaje depende cada vez más de las interacciones. Se promueve la ayuda mutua y el principio de solidaridad (FLECHA, 2000). En este sentido, la diversidad entre el alumnado se entiende como un factor positivo para el aprendizaje, y los estudiantes encuentran el apoyo que necesitan realizando actividades con otras personas que tienen distintas habilidades o que tienen mayor capacidad en determinadas materias y en otras no. Esta AEE proporciona un espacio de interacción con miembros de la comunidad y personas adultas que no pertenecen al ámbito de estudio; en cada GI hay personas voluntarias que facilitan la dinamización del diálogo y las interacciones del alumnado con las actividades. De esta forma dan soporte directo a los estudiantes y colaboran alentando la participación y el apoyo mutuo entre los grupos. Los niños encuentran la oportunidad de recibir ayuda desde sus pares, así como de las 
personas adultas voluntarias, y esto ocurre como parte de un desarrollo regular dentro de la actividad, logrando mayor inclusión social y otorgando mayores oportunidades al alumnado, prestando atención a niños con necesidades específicas de apoyo educativo (NEAE) que en otros contextos tienden a ser excluidos, o atribuir menores expectativas acerca de sus resultados académicos. La atención a las necesidades especiales desde las AEE toma de referencia no sólo al individuo, sino también el contexto donde éste aprende y se desarrolla. Esta perspectiva es la que permite responder de manera más efectiva a las NEAE, aumentando las expectativas y evitando la reducción del currículum. De este modo la organización que se realiza en el aula es inclusiva, permite la participación del voluntariado, exalumnos, familiares y personas de la comunidad. Logra multiplicar y diversificar las interacciones, a la vez que aumenta el tiempo de trabajo efectivo; este tipo de actuación evita la segregación que se genera al dividir al alumnado con necesidades diferenciales o dificultades de aprendizaje, lo que problematiza el correcto desempeño e integración con el grupo de estudio. Los GI rechazan la aplicación de adaptaciones curriculares que han generado mayores tasas de fracaso escolar y conflictos, por la falta de integración y discriminación que se genera por este tipo de prácticas segregadoras y se diferencia claramente de la organización mixta o streaming. Si queremos mejorar el aprendizaje y la calidad educativa de los niños debemos transformar las interacciones y la modalidad de organización en el trabajo en el aula.

Diversas investigaciones han demostrado que la agrupación por grupos con dificultades es asociada con bajos niveles de capacidad, baja seguridad de sus competencias y resultados académicos, baja autoestima y expectativas, y bajo rendimiento (DunN, 1968; FITCH, 2003, FISHER, ROACH y FrEY, 2002). El sistema de división por aptitudes (tracking o streaming) se caracteriza por generar distintos itinerarios educativos, por el incremento de los recursos humanos y la separación del alum-

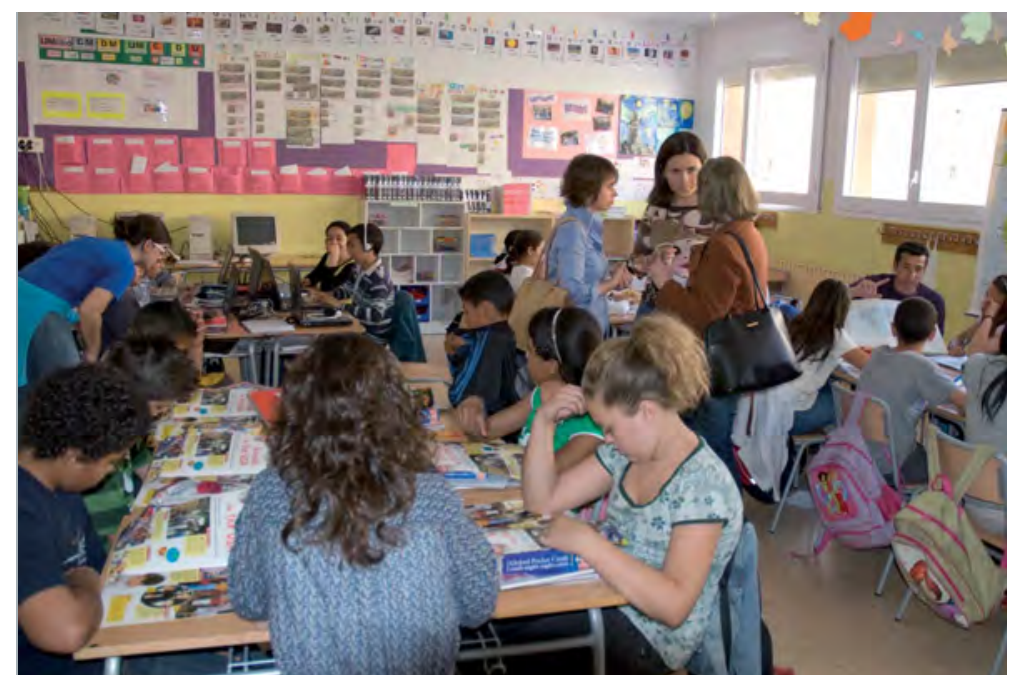

Figura 1. Organización de los grupos interactivos

\section{Actividades cortas} que se completan aproximadamente en 20 minutos

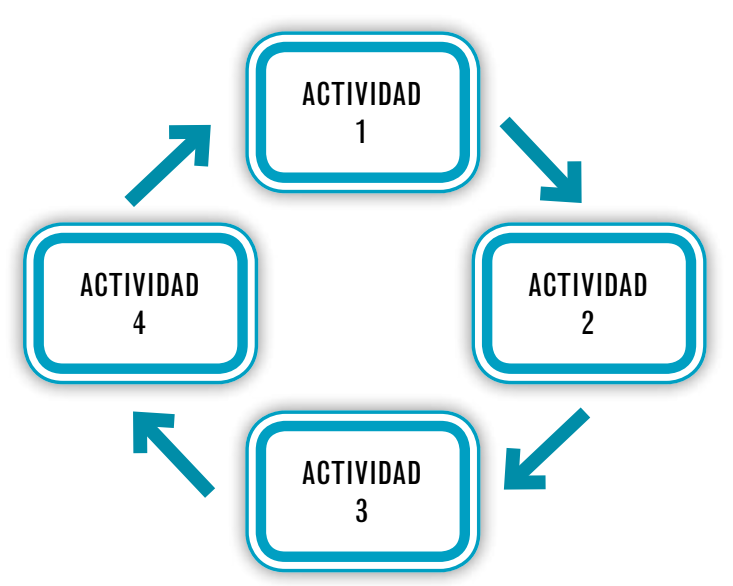

nado etiquetado como diferente o difícil a un aula o centro. Tiene como resultado un impacto negativo en el rendimiento escolar y sobre las futuras oportunidades en el alumnado, marcando desigualdades por el modo de agrupamiento y aumenta la posibilidad de futura exclusión (FLECHA, 2015). Esta modalidad de agrupación afecta principalmente a los grupos vulnerables con bajo nivel socioeconómico, donde los niveles de exigencia académica suelen ser menores, donde ven reducido el horario destinado a actividades de aprendizaje y reciben menos estimulación para la reflexión y el pensamiento crítico (CHORzEMPA y GRAHAM, 2006). El streaming reduce el efecto que tienen los compañeros con un mejor rendimiento sobre la mejora del aprendizaje del alumnado con rendimiento medio y bajo, al no poder interaccionar conjuntamente (ZIMMER, 2003). También aumentan los problemas de convivencia, 


\section{IIII. ÁQORA DE DROFESORES}

¿QUÉ ES?
Una forma de organización del aula.
$\begin{aligned} & \text { Grupos reducidos de alumnado agrupados de } \\ & \text { forma heterogénea de niveles de aprendizaje, } \\ & \text { cultura, género, etc. }\end{aligned}$

Grupos donde se establecen relaciones entre los y las alumnas que forman parte del grupo por medio del diálogo igualitario.

Cada grupo cuenta con la presencia de un adulto referente que puede ser el maestro, familiares, u otros voluntarios. El aprendizaje de los alumnos y alumnas depende cada vez más del conjunto de sus interacciones y no sólo de las que se producen en el aula tradicional.

La participación de las personas voluntarias en el aula facilita el aprendizaje y aumenta la motivación de los niños y niñas por el aprendizaje, creando un buen clima de trabajo.

Todos los niños y niñas del grupo trabajan sobre la misma tarea.

Tanto el profesorado como las personas voluntarias mantienen unas altas expectativas hacia el alumnado.

Todos los niños y niñas aprenden, incluso aquellos que tienen facilidad, porque ayudar al otro, implica un ejercicio de metacognición que contribuye a consolidar los conocimientos, hasta el punto de ser capaz de explicarlos a otras personas.

\section{¿QUÉ NO ES?}

Una metodología.

Grupos cooperativos

Agrupaciones flexibles.

Dividir la clase por grupos con un solo adulto como referente, el profesor.

Si previamente se ha sacado de aula al alumnado con bajo nivel de aprendizaje.

Si, sin sacar a nadie del aula, los grupos se forman de forma homogénea de acuerdo con el nivel de aprendizaje.

Si se hacen agrupaciones heterogéneas en un aula donde está el alumnado de mayor nivel de aprendizaje.

Si dentro de los grupos interactivos heterogéneos se dan tareas diferentes a los niños y niñas por nivel de aprendizaje.

Si no hay interacción entre los alumnos mientras se resuelve la tarea planteada. por lo que países como Finlandia, con mejores resultados académicos, prohibió por ley en los años 80 esta práctica en las escuelas (INCLUD-ED, 2011).

Otro tipo de formación con bajo impacto es mixture, el modo de organización del aula tradicional, en la que se incluye a todo el alumnado, con un profesor. Si bien este tipo de formación del aula tiene como principio la igualdad, la aplicación de este tipo de prácticas tiene dificultades a la hora de responder a las necesidades de los niños con situaciones vulnerables y dar respuesta a la diversidad del alumnado (VAlls, Siles y Molina, 2011). Las características de la formación son las siguientes: todo el alumnado en su conjunto en la misma aula y un sólo profesor, lo que no ofrece una respuesta adecuada a la diversidad de necesidades del alumnado, presentando serios problemas para conseguir el éxito educativo en los rendimientos académicos.

El aprendizaje dialógico es la base de los grupos interactivos (AUBERT et al., 2008). Se construyen con el diálogo igualitario e interacciones horizontales entre las personas, y centra su atención en los vínculos que se establecen dentro de la escuela y fuera del aula, con miembros de la comunidad, incluyendo todas las voces. Vygotsky (1979) elaboró la teoría de que los niños pueden aprender más a través de las interacciones que en un estudio individual aislado. Según Vygotsky, las interacciones sociales en el proceso de aprendizaje tienen un impacto en el desarrollo de los procesos elementales (biológicos) y en los procesos psicológicos superiores. Freire (1997) defendió la posibilidad de transformar el contexto de aprendizaje a través de la acción humana y subrayó el poder de la educación para superar las desigualdades sociales. Es así que las interacciones humanas tienen un papel central en el desarrollo de las capacidades intelectuales y el rendimiento académico. Bruner (1997) enfatizaba la posibilidad de aprender a través de interacciones de los estudiantes, ya sea a través de la enseñanza de cada uno o de los vínculos con otros. En la teoría de la acción comunicativa, Habermas (1987) conceptualiza la acción comunicativa racional como la racionalidad que utiliza el conocimiento para lograr un acuerdo sobre una determinada tarea, y la acción comunicativa como la acción que tiene lugar cuando los actores tienen que estar de acuerdo en una situación o plan de acción que tienen que llevar a cabo para resolver un problema. De acuerdo 
Figura 2. Composición de los grupos interactivos

con Habermas todas las personas tienen la capacidad de lenguaje y acción, y por lo tanto todas las personas pueden tener lugar en las acciones comunicativas y acciones de concertación para llegar al consenso. Las interacciones dan proximidad a la explicación, desarrollan habilidades comunicativas, generando múltiples procesos congnitivos. De esta forma, se consolida lo aprendido, aumentan los niveles de comprensión y aprendizaje.

Es así que las AEE responden a la necesidad de cambio del sistema educativo, en un contexto de diversidad y un mundo cada vez más conectado y exigente académicamente, que necesita responder ante la pluralidad de situaciones y desigualdades del alumnado. Tienen el objetivo de dar soporte a todo el alumnado para lograr el éxito educativo y asegurar su inclusión social, buscando así la equidad a través de la diversidad y no dentro de un contexto homogéneo, que no es representante de las sociedades actuales. Por tanto, contemplan la heterogeneidad, en términos culturales, étnicos, de género, de habilidades, en vista de la aplicación del principio de equidad de diferencias (FLECHA, 2000). En este contexto, las distintas identidades son respetadas y valuadas, entendiendo que todos los estudiantes ofrecen un aporte relevante para lograr el éxito educativo mediante un diálogo participativo e igualitario que busca la superación y máximas expectativas para el alumnado.

Los resultados de la aplicación se reflejan en la aceleración de los aprendizajes, el cambio en la dinámica en los conflictos, y en la mejora de la relación entre el alumnado y la escuela. Se realzan los valores de solidaridad y socialización preventiva para la violencia, así como un interés en aprender y mejorar la vida y la calidad de la educación. Son muchas las escuelas que experimentan en poco tiempo un impacto muy potente en el aprendizaje, inducción de la ayuda, valoración de la diversidad, altas expectativas en el alumnado, profesorado y familiares, así como mayor participación con más confianza, mejora del ambiente y convivencia y reconocimiento de las ca-

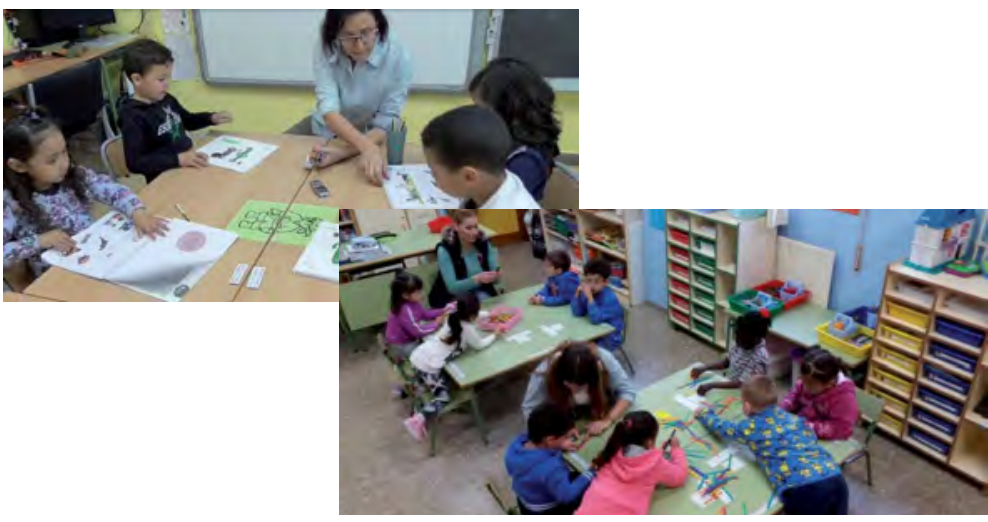

Alumnos que trabajan agrupados en 4 grupos heterogéneos con un adulto en cada grupo.

pacidades de los niños con necesidades específicas de apoyo educativo, donde se destaca el éxito, percibiendo los beneficios para sus compañeros y para la comunidad, potenciando los sentimientos y la amistad. Y con todo ello la solidaridad entre ellos

\section{DARA SABER MÁS}

AA.vv. (2012). Claves para conseguir el éxito educativo. Cuadernos de Pedagogía, 429. Número Monográfico.

InClud-ed Consortium (2011). Actuaciones de éxito en las escuelas europeas. Madrid: Ministerio de Educación IFIIE. Publicación basada en los resultados finales del proyecto del VI Programa Marco INCLUD-ED. Recuperado de http://utopiadream.info/ca/ wp-content/uploads/2011/04/Actuaciones-de-\%C3\%A9xito-enlas-escuelas-europeas.pdf

VALLS, R., \& KYRIAKIDES, L. (2013). The power of interactive groups: how diversity of adults volunteering in classroom groups can promote inclusion and success for children of vulnerable minority ethnic populations. Cambridge Journal of Education, 43 (1), 17-33. doi: 10.1080/0305764X.2012.749213

\section{HEMOS HABLADO DE}

\section{Organización en el aula; aprendizaje dialógico; grupos interactivos; solidaridad; Comunidades de aprendizaje.}

Este artículo fue solicitado por PADRES y MAESTROS en diciembre de 2015, revisado y aceptado en julio de 2016. 